\title{
NOTES ON THE FROBENIUS TEST EXPONENTS
}

\author{
DUONG THI HUONG AND PHAM HUNG QUY
}

\begin{abstract}
In this paper we show that the Frobenius test exponent for parameter ideals of a local ring of prime characteristic is always bigger than or equal to its Hartshorne-SpeiserLyubeznik number. Our argument is based on an isomorphism of Nagel and Schenzel on local cohomology for which we will provide an elementary proof.
\end{abstract}

\section{INTRODUCTION}

Let $R$ be a Noetherian commutative ring of prime characteristic $p>0$, and $I$ an ideal of $R$. The Frobenius closure of $I$ is $I^{F}=\left\{x \mid x^{p^{e}} \in I^{\left[p^{e}\right]}\right.$ for some $\left.e \geq 0\right\}$, where $I^{\left[p^{e}\right]}=\left(r^{p^{e}} \mid r \in I\right)$ is the $e$-th Frobenius power of $I$. It is hard to compute $I^{F}$. By the Noetherianess of $R$ there is an integer $e$, depending on $I$, such that $\left(I^{F}\right)^{\left[p^{e}\right]}=I^{\left[p^{e}\right]}$. We call the smallest number $e$ satisfying the condition the Frobenius test exponent of $I$, and denote it by Fte $(I)$. It is natural to expect the existence of a uniform number $e$, depending only on the ring $R$, such that, for every ideal $I$ we have $\left(I^{F}\right)^{\left[p^{e}\right]}=I^{\left[p^{e}\right]}$, i.e. $F t e(I) \leq e$ for every ideal $I$. If we have a positive answer to this question, then the two conditions $x \in I^{F}$ and $x^{p^{e}} \in I^{\left[p^{e}\right]}$ are equivalent. This gives in particular a finite test for the Frobenius closure. We call such a number $e$ a Frobenius test exponent for the ring $R$. However, Brenner 2] gave two-dimensional normal standard graded domains with no Frobenius test exponent. In contrast, Katzman and Sharp [8] showed the existence of a uniform bound of Frobenius test exponents if we restrict to the class of parameter ideals in a Cohen-Macaulay local ring. For any local ring $(R, \mathfrak{m})$ we define the Frobenius test exponent for parameter ideals, denoted by Fte $(R)$, is the smallest integer $e$ such that $\left(\mathfrak{q}^{F}\right)^{\left[p^{e}\right]}=\mathfrak{q}^{\left[p^{e}\right]}$ for every parameter ideal $\mathfrak{q}$ of $R$, and Fte $(R)=\infty$ if we have no such integer. Katzman and Sharp asked whether $\operatorname{Fte}(R)<\infty$ for any (equidimensional) local ring. Furthermore, the authors of [7] confirmed the question for generalized CohenMacaulay local rings. Recently the second author gave a positive answer for the question for $F$-nilpotent rings [14].

The main idea in [7, 8] is connecting the Frobenius test exponent for parameter ideals with an invariant defined by the Frobenius actions on the local cohomology modules $H_{\mathfrak{m}}^{i}(R)$, namely the Hartshorne-Speiser-Lyubeznik number of $H_{\mathfrak{m}}^{i}(R)$. Recall that the Frobenius endomorphism $F: R \rightarrow R, x \mapsto x^{p}$ induces Frobenius actions on the local cohomology modules $H_{\mathfrak{m}}^{i}(R)$ for all $i \geq 0$. Roughly speaking, the Hartshorne-Speiser-Lyubeznik number of $H_{\mathfrak{m}}^{i}(R)$, denoted by $H S L\left(H_{\mathfrak{m}}^{i}(R)\right)$, is a nilpotency index of Frobenius actions on $H_{\mathfrak{m}}^{i}(R)$

Key words and phrases. The Frobenius test exponent, The Hartshorne-Speiser-Lyubeznik number, Local cohomology, Filter regular sequence.

2010 Mathematics Subject Classification:13A35, 13D45.

The second author is partially supported by a fund of Vietnam National Foundation for Science and Technology Development (NAFOSTED) under grant number 101.04-2017.10. 
for all $i \geq 0$ (see Section 3 for details). The Hartshorne-Speiser-Lyubeznik number of $R$ is $H S L(R)=\max \left\{H S L\left(H_{\mathfrak{m}}^{i}(R)\right) \mid i=0, \ldots, \operatorname{dim} R\right\}$. Moreover, the Frobenius action $F: H_{\mathfrak{m}}^{i}(R) \rightarrow H_{\mathfrak{m}}^{i}(R)$ is injective for all $i \geq 0$ (in this case, $R$ is called $F$-injective) if and only if $H S L(R)=0$. If $R$ is Cohen-Macaulay, Katzman and Sharp proved the equality $\operatorname{Fte}(R)=H S L(R)$. The main result of the present paper as follows

Theorem 1.1. Let $(R, \mathfrak{m})$ be a local ring of positive characteristic $p>0$ of dimension $d$. Then Fte $(R) \geq H S L(R)$.

Our main technique is to analyze the local cohomology modules by using the NagelSchenzel isomorphism (cf. [10, Proposition 3.4]). Then we can consider any local cohomology $H_{\mathfrak{m}}^{i}(R)$ as a submodule of a top local cohomology whose Frobenius action can be understood explicitly. In the next section, we will give a new and simple proof for Nagel-Schenzel's isomorphism. The main result will be proved in Section 3 (Theorem 3.4). We also prove that the Frobenius test exponent for parameter ideals has a good behavior under localization (Proposition 3.5).

\section{NAGEL-SCHENZEL'S ISOMORPHISM}

In this section, let $R$ be a commutative Noetherian ring, $M$ a finitely generated $R$-module and $I$ an ideal of $R$. The use of $I$-filter regular sequences on $M$ provide an useful technique for the study of local cohomology. In [10, Proposition 3.4] Nagel and Schenzel proved the following useful theorem (see also [1]).

Theorem 2.1. Let I be an ideal of a Noetherian ring $R$ and $M$ a finitely generated $R$-module. Let $x_{1}, \ldots, x_{t}$ an $I$-filter regular sequence of $M$. Then we have

$$
H_{I}^{i}(M) \cong \begin{cases}H_{\left(x_{1}, \ldots, x_{t}\right)}^{i}(M) & \text { if } i<t \\ H_{I}^{i-t}\left(H_{\left(x_{1}, \ldots, x_{t}\right)}^{t}(M)\right) & \text { if } i \geq t .\end{cases}
$$

The most important case of Theorem 2.1 is $i=t$, and so $H_{I}^{t}(M) \cong H_{I}^{0}\left(H_{\left(x_{1}, \ldots, x_{t}\right)}^{t}(M)\right)$ a submodule of $H_{\left(x_{1}, \ldots, x_{t}\right)}^{t}(M)$. Recently, many applications of this fact have been found [4, 12, 13. It should be noted that Nagel-Schenzel's theorem was proved by using spectral sequences. The aim of this section is to give an elementary proof for Theorem [2.1] based on standard arguments of local cohomology [3]. We recall the definition and some simple properties of $I$-filter regular sequences.

Definition 2.2. Let $M$ be a finitely generated module $R$ and let $x_{1}, \ldots, x_{t} \in I$ be a sequence of elements of $R$. Then we say that $x_{1}, \ldots, x_{t}$ is a $I$-filter regular sequence on $M$ if the following condition hold:

$$
\operatorname{Supp}\left(\left(\left(x_{1}, \ldots, x_{i-1}\right) M: x_{i}\right) /\left(x_{1}, \ldots, x_{i-1}\right) M\right) \subseteq V(I)
$$

for all $i=1, \ldots, t$, where $V(I)$ denotes the set of prime ideals containing $I$. This condition is equivalent to $x_{i} \notin \mathfrak{p}$ for all $\mathfrak{p} \in \operatorname{Ass}_{R} M /\left(x_{1}, \ldots, x_{i-1}\right) M \backslash V(I)$ and for all $i=1, \ldots, t$. In the case $(R, \mathfrak{m})$ is a local ring, we call an $\mathfrak{m}$-filter regular sequence of $M$ simply by a filter regular sequence of $M$. 
Remark 2.3. It should be noted that for any $t \geq 1$ we always can choose a $I$-filter regular sequence $x_{1}, \ldots, x_{t}$ on $M$. Indeed, by the prime avoidance lemma we can choose $x_{1} \in I$ and $x_{1} \notin \mathfrak{p}$ for all $\mathfrak{p} \in \operatorname{Ass}_{R} M \backslash V(I)$. For $i>1$ assume that we have $x_{1}, \ldots, x_{i-1}$, then we choose $x_{i} \in I$ and $x_{i} \notin \mathfrak{p}$ for all $\mathfrak{p} \in \operatorname{Ass}_{R} M /\left(x_{1}, \ldots, x_{i-1}\right) M \backslash V(I)$ by the prime avoidance lemma again. For more details, see [1, Section 2].

The $I$-filter regular sequence can be seen as a generalization of the well-known notion of regular sequences (cf. [10, Proposition 2.2]).

Lemma 2.4. A sequence $x_{1}, \ldots, x_{t} \in I$ is an $I$-filter regular sequence on $M$ if and only if for all $\mathfrak{p} \in \operatorname{Supp}(M) \backslash V(I)$, and for all $i \leq t$ such that $x_{1}, \ldots, x_{i} \in \mathfrak{p}$ we have $\frac{x_{1}}{1}, \cdots, \frac{x_{i}}{1}$ is an $M_{\mathfrak{p}}$-sequence.

Corollary 2.5. Let $x_{1}, \ldots, x_{t} \in I$ be an $I$-filter regular sequence on $M$. Then $H_{\left(x_{1}, \ldots, x_{t}\right)}^{i}(M)$ is $I$-torsion for all $i<t$.

Proof. For each $\mathfrak{p} \in \operatorname{Supp}(M) \backslash V(I)$ we have either $\left(x_{1}, \ldots x_{t}\right) R_{\mathfrak{p}}=R_{\mathfrak{p}}$ or $x_{1}, \ldots, x_{t}$ is an $M_{\mathfrak{p}}$-regular sequence by Lemma 2.4. For the first case we have

$$
\left(H_{\left(x_{1}, \ldots, x_{t}\right)}^{i}(M)\right)_{\mathfrak{p}} \cong H_{\left(x_{1}, \ldots, x_{t}\right) R_{\mathfrak{p}}}^{i}\left(M_{\mathfrak{p}}\right)=0
$$

for all $i \geq 0$. For the second case we have

$$
\left(H_{\left(x_{1}, \ldots, x_{t}\right)}^{i}(M)\right)_{\mathfrak{p}} \cong H_{\left(x_{1}, \ldots, x_{t}\right) R_{\mathfrak{p}}}^{i}\left(M_{\mathfrak{p}}\right)=0
$$

for all $i<t$ by the Grothendieck vanishing theorem [3, Theorem 6.2.7]. Therefore we have $\left(H_{\left(x_{1}, \ldots, x_{t}\right)}^{i}(M)\right)_{\mathfrak{p}} \cong 0$ for all $i<t$ and for all $\mathfrak{p} \in \operatorname{Spec}(R) \backslash V(I)$. So $H_{\left(x_{1}, \ldots, x_{t}\right)}^{i}(M)$ is $I$-torsion for all $i<t$.

It is well-known that local cohomology $H_{\left(x_{1}, \ldots, x_{t}\right)}^{i}(M)$ agrees with the $i$-th cohomology of the Cech complex with respect to the sequence $x_{1}, \ldots, x_{t}$

$$
0 \rightarrow M \stackrel{d^{0}}{\longrightarrow} \bigoplus_{i} M_{x_{i}} \stackrel{d^{1}}{\longrightarrow} \bigoplus_{i<j} M_{x_{i} x_{j}} \stackrel{d^{2}}{\longrightarrow} \cdots \stackrel{d^{t-1}}{\longrightarrow} M_{x_{1} \ldots x_{t}} \rightarrow 0 .
$$

The following simple fact plays an important role in our proof.

Lemma 2.6. Let $x \in I$ be any element of $R$ and $M$ an $R$-module. Then $H_{I}^{i}\left(M_{x}\right)=0$ for all $i \geq 0$.

Proof. The multiplication map $M_{x} \stackrel{x}{\rightarrow} M_{x}$ is an isomorphism. It induces isomorphism maps $H_{I}^{i}\left(M_{x}\right) \stackrel{x}{\rightarrow} H_{I}^{i}\left(M_{x}\right)$ for all $i \geq 0$. But $H_{I}^{i}\left(M_{x}\right)$ is $I$-torsion, so it is $(x)$-torsion since $x \in I$. Therefore $H_{I}^{i}\left(M_{x}\right)=0$ for all $i \geq 0$.

By Corollary 2.5 and Lemma 2.6 the theorem of Nagel and Schenzel is a special case of the following theorem.

Theorem 2.7. Let $I$ be an ideal of a Noetherian ring $R$ and $M$ an $R$-module. Suppose we have a complex of $R$-modules

$$
0 \rightarrow M=M^{0} \stackrel{d^{0}}{\rightarrow} M^{1} \stackrel{d^{1}}{\rightarrow} M^{2} \stackrel{d^{2}}{\rightarrow} \cdots \stackrel{d^{t-1}}{\rightarrow} M^{t} \rightarrow 0, \quad(\star)
$$


where $H_{I}^{j}\left(M^{i}\right)=0$ for all $i>0$ and for all $j \geq 0$. Suppose that the cohomology $H^{i}:=$ $\operatorname{Ker}\left(d^{i}\right) / \operatorname{Im}\left(d^{i-1}\right)$ is I-torsion for all $i<t$. Then we have the following isomorphism

$$
H_{I}^{i}(M) \cong \begin{cases}H^{i} & \text { if } i<t \\ H_{I}^{i-t}\left(H^{t}\right) & \text { if } i \geq t .\end{cases}
$$

In particular, if $H^{t}$ is also $I$-torsion then $H_{I}^{i}(M) \cong H^{i}$ for all $i \geq 0$.

Proof. It is sufficient to prove the first assertion. For all $j \geq 0$ we set $L^{j}:=\operatorname{Im}\left(d^{j-1}\right)$ and $K^{j}:=\operatorname{Ker}\left(d^{j}\right)$. Hence $H^{j} \cong K^{j} / L^{j}$ for all $j \geq 0$. We split the complex $(\star)$ into short exact sequences

$$
\begin{gathered}
0 \rightarrow L^{t-1} \rightarrow K^{t-1} \rightarrow H^{t-1} \rightarrow 0 \\
0 \rightarrow K^{t-1} \rightarrow M^{t-1} \rightarrow L^{t} \rightarrow 0 \\
0 \rightarrow L^{t} \rightarrow M^{t} \rightarrow H^{t} \rightarrow 0 .
\end{gathered}
$$

Since $L^{j}$ and $K^{j}$ are submodules of $M^{j}$ for all $j \geq 1$, we have $H_{I}^{0}\left(L^{j}\right) \cong H_{I}^{0}\left(K^{j}\right)=0$ for all $j \geq 1$. We also note that $H^{j}$ is $I$-torsion for all $j<t$ by the assumption, so $H_{I}^{0}\left(H^{j}\right)=H^{j}$ and $H_{I}^{i}\left(H^{j}\right) \cong 0$ for all $j<t$ and for all $i \geq 1$.

Now applying the functor $H_{I}^{i}(-)$ to the short exact sequence $\left(A_{0}\right)$ and using the above observations we have

$$
H_{I}^{0}(M) \cong H^{0}
$$

and

$$
H_{I}^{i}(M) \cong H_{I}^{i}\left(L^{1}\right)
$$

for all $i \geq 1$.

For each $j=1, \ldots, t-1$, applying the local cohomology functor $H_{I}^{i}(-)$ to the short exact sequence $\left(A_{j}\right)$ we have $H_{I}^{1}\left(K^{j}\right) \cong 0$ and the isomorphism

$$
H_{I}^{i}\left(L^{j+1}\right) \cong H_{I}^{i+1}\left(K^{j}\right)
$$

for all $i \geq 1$. Furthermore, if we apply $H_{I}^{i}(-)$ for the short exact sequence $\left(B_{j}\right)$, then we obtain the short exact sequence

$$
0 \rightarrow H_{I}^{0}\left(H^{j}\right) \cong H^{j} \rightarrow H_{I}^{1}\left(L^{j}\right) \rightarrow H_{I}^{1}\left(K^{j}\right) \rightarrow 0,
$$


and the isomorphism

$$
H_{I}^{i}\left(L^{j}\right) \cong H_{I}^{i}\left(K^{j}\right)
$$

for all $i \geq 2$. Note that $H_{I}^{1}\left(K^{j}\right)=0$ as above, so

$$
H^{j} \cong H_{I}^{1}\left(L^{j}\right) \text {. }
$$

By the isomorphisms $\left(C_{j}\right)$ and $\left(D_{j}\right)$ we have $H_{I}^{i}\left(L^{j+1}\right) \cong H_{I}^{i+1}\left(L^{j}\right)$ for all $j=1, \ldots, t-1$ and for all $i \geq 1$. We next show that $H_{I}^{i}(M) \cong H^{i}$ for all $i=1, \ldots, t-1$. Indeed, using isomorphisms (1),(2) and the above isomorphism we have

$$
H_{I}^{i}(M) \stackrel{(1)}{\cong} H_{I}^{i}\left(L^{1}\right) \cong H_{I}^{i-1}\left(L^{2}\right) \cong \cdots \cong H_{I}^{1}\left(L^{i}\right) \stackrel{(2)}{\cong} H^{i}
$$

Therefore, we have showed the isomorphisms $H_{I}^{i}(M) \cong H^{i}$ for all $i=0, \ldots, t-1$. Finally, for $i \geq t$ by similar arguments we have

$$
H_{I}^{i}(M) \stackrel{(1)}{\cong} H_{I}^{i}\left(L^{1}\right) \cong H_{I}^{i-1}\left(L^{2}\right) \cong \cdots \cong H_{I}^{i-t+1}\left(L^{t}\right)
$$

On the other hand, by applying the functor $H_{I}^{i}(-)$ to the short exact sequence $\left(B_{t}\right)$ we have

$$
H_{I}^{i-t}\left(H^{t}\right) \cong H_{I}^{i-t+1}\left(L^{t}\right)
$$

for all $i \geq t$. Thus $H_{I}^{i}(M) \cong H_{I}^{i-t}\left(H^{t}\right)$ for all $i \geq t$, and we finish the proof.

Remark 2.8. Let $I=\left(x_{1}, \ldots, x_{t}\right)$ be an ideal of $R$. It is not hard to show that the cohomology of Čech complex $\breve{C}\left(x_{1}, \ldots, x_{t} ; M\right)$ is always $I$-torsion for any $R$-module $M$. By the last assertion of Theorem 2.7 we obtain the well-known fact $H_{I}^{i}(M) \cong H^{i}\left(\breve{C}\left(x_{1}, \ldots, x_{t} ; M\right)\right)$ for all $i \geq 0$.

\section{On the Frobenius test EXPONENT FOR PARAMETER IDEALS}

In this section, let $R$ be a Noetherian ring containing a field of characteristic $p>0$. Let $F: R \rightarrow R, x \mapsto x^{p}$ denote the Frobenius endomorphism. If we want to notationally distinguish the source and target of the $e$-th Frobenius endomorphism $F^{e}: R \stackrel{x \mapsto x^{p^{e}}}{\longrightarrow} R$, we will use $F_{*}^{e}(R)$ to denote the target. $F_{*}^{e}(R)$ is an $R$-bimodule, which is the same as $R$ as an abelian group and as a right $R$-module, that acquires its left $R$-module structure via the $e$-th Frobenius endomorphism $F^{e}$. By definition the $e$-th Frobenius endomorphism $F^{e}: R \rightarrow F_{*}^{e}(R)$ sending $x$ to $F_{*}^{e}\left(x^{p^{e}}\right)=x \cdot F_{*}^{e}(1)$ is an $R$-homomorphism.

Definition $3.1([6])$. Let $I$ be an ideal of $R$ we define

(1) The $e$-th Frobenius power of $I$ is $I^{\left[p^{e}\right]}=\left(x^{p^{e}} \mid x \in I\right)$.

(2) The Frobenius closure of $I, I^{F}=\left\{x \mid x^{p^{e}} \in I^{\left[p^{e}\right]}\right.$ for some $\left.e \geq 0\right\}$.

Remark 3.2. An element $x \in I^{F}$ if it is contained in the kernel of the composition

$$
R \rightarrow R / I \cong R / I \otimes_{R} R \stackrel{\mathrm{id} \otimes F^{e}}{\longrightarrow} R / I \otimes_{R} F_{*}^{e}(R)
$$

for some $e \geq 0$. Moreover $R$ is Noetherian, so $I^{F}$ is finitely generated. Therefore there exists an integer $e_{0}$ such that

$$
I^{F}=\operatorname{Ker}\left(R \rightarrow R / I \cong R / I \otimes_{R} R \stackrel{\operatorname{id} \otimes F^{e}}{\longrightarrow} R / I \otimes_{R} F_{*}^{e}(R)\right)
$$


for all $e \geq e_{0}$.

By the above discussion for every ideal $I$ there is an integer $e$ (depending on $I$ ) such that $\left(I^{F}\right)^{\left[p^{e}\right]}=I^{\left[p^{e}\right]}$. A problem of Katzman and Sharp [8, Introduction] asks in its strongest form: does there exist a number $e$, depending only on the $\operatorname{ring} R$, such that, for every ideal $I$ we have $\left(I^{F}\right)^{\left[p^{e}\right]}=I^{\left[p^{e}\right]}$. A positive answer to this question, together with the actual knowledge of a bound for $e$, would give an algorithm to compute the Frobenius closure $I^{F}$. We call such a number $e$ a Frobenius test exponent for the $\operatorname{ring} R$. Unfortunately, Brenner [2] gave twodimensional normal standard graded domains with no Frobenius test exponent. In contrast, Katzman and Sharp showed the existence of Frobenius test exponent if we restrict to class of parameter ideals in a Cohen-Macaulay ring. It leads the following question.

Question 1. Let $(R, \mathfrak{m})$ be an (equidimensional) local ring of prime characteristic $p$. Then does there exist an integer e such that for every parameter ideal $\mathfrak{q}$ of $R$ we have $\left(\mathfrak{q}^{F}\right)^{\left[p^{e}\right]}=\mathfrak{q}^{\left[p^{e}\right]}$ ?

We definite the Frobenius test exponent for parameter ideals of $R, F t e(R)$, the smallest integer $e$ satisfying the above condition and $F t e(R)=\infty$ if we have no such $e$. Question 1 has affirmative answers when $R$ is either generalized Cohen-Macaulay by [7] or $F$-nilpotent by [14], and is open in general. The Frobenius test exponent for parameter ideals is closely related with an invariant defined in terms of Frobenius action on local cohomology. For any ideal $I=\left(x_{1}, \ldots, x_{t}\right)$, the Frobenius endomorphism $F: R \rightarrow R$ and its localizations induce a natural Frobenius action on local cohomology $F: H_{I}^{i}(R) \rightarrow H_{I[p]}^{i}(R) \cong H_{I}^{i}(R)$ for all $i \geq 0$. There is a very useful way of describing the top local cohomology. It can be given as the direct limit of Koszul cohomologies

$$
H_{I}^{t}(R) \cong \lim _{\longrightarrow} R /\left(x_{1}^{n}, \ldots, x_{t}^{n}\right)
$$

with the map in the system $\varphi_{n, m}: R /\left(x_{1}^{n}, \ldots, x_{t}^{n}\right) \rightarrow R /\left(x_{1}^{m}, \ldots, x_{t}^{m}\right)$ is multiplication by $\left(x_{1} \ldots x_{t}\right)^{m-n}$ for all $m \geq n$. Then for each $\bar{a} \in H_{I}^{t}(R)$, which is the canonical image of $a+\left(x_{1}^{n}, \ldots, x_{t}^{n}\right)$, we find that $F(\bar{a})$ is the canonical image of $a^{p}+\left(x_{1}^{p n}, \ldots, x_{t}^{p n}\right)$.

Notice that $H_{\mathfrak{m}}^{i}(R)$ is always Artinian for all $i \geq 0$. Let $A$ be an Artinian $R$-module with a Frobenius action $F: A \rightarrow A$. Then we define the Frobenius closure $0_{A}^{F}$ of the zero submodule of $A$ is the submodule of $A$ consisting all elements $z$ such that $F^{e}(z)=0$ for some $e \geq 0.0_{A}^{F}$ is the nilpotent part of $A$ by the Frobenius action. By [5, Proposition 1.11] and [9, Proposition 4.4] there exists a non-negative integer $e$ such that $0_{A}^{F}=\operatorname{Ker}\left(A \stackrel{F^{e}}{\longrightarrow} A\right.$ ) (see also [15]). The smallest of such integers is called the Hartshorne-Speiser-Lyubeznik number of $A$ and denoted by $H S L(A)$. We define the Hartshorne-Speiser-Lyubeznik number of a local ring $(R, \mathfrak{m})$ as follows

$$
H S L(R):=\min \left\{e \mid 0_{H_{\mathfrak{m}}^{i}(R)}^{F}=\operatorname{Ker}\left(H_{\mathfrak{m}}^{i}(R) \stackrel{F^{e}}{\longrightarrow} H_{\mathfrak{m}}^{i}(R)\right) \text { for all } i=0, \ldots, d\right\} .
$$

If $R$ is Cohen-Macaulay, then Katzman and Sharp [8] showed that Fte $(R)$ is just HSL $(R)$. In this paper we will show that $F t e(R) \geq H L S(R)$ for any local ring $R$. We need the following result.

Proposition 3.3. Let $x_{1}, \ldots, x_{t}$ be a sequence of elements in $R$. Then we have

$$
0_{H_{(\underline{x})}^{t}(R)}^{F} \cong \underset{n}{\lim } \frac{\left(x_{1}^{n}, \ldots, x_{t}^{n}\right)^{F}}{\left(x_{1}^{n}, \ldots, x_{t}^{n}\right)} .
$$


Proof. For each $e \geq 0$ the Frobenius action $F^{e}$ on $H_{(\underline{x})}^{t}(R)$ is the direct limit of the following commutative diagram:

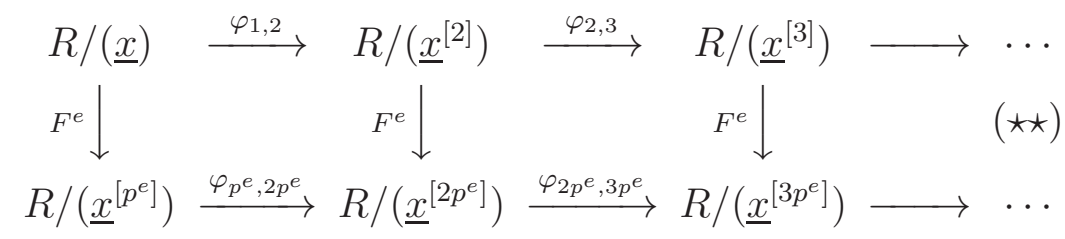

where each vertical map is the Frobenius homomorphism. For each $a \in\left(x_{1}^{n}, \ldots, x_{t}^{n}\right)^{F}$, it is clear that $a+\left(x_{1}^{n}, \ldots, x_{t}^{n}\right)$ maps to an element in $0_{H_{(\underline{x})}^{t}}^{F}(R)$. Thus we have an injection

$$
\underset{n}{\lim } \frac{\left(x_{1}^{n}, \ldots, x_{t}^{n}\right)^{F}}{\left(x_{1}^{n}, \ldots, x_{t}^{n}\right)} \hookrightarrow 0_{H_{(\underline{x})}^{t}(R)}^{F} .
$$

For the surjection, let $\bar{a}$ be any element of $0_{H_{(\underline{x})}^{t}}^{F}(R)$. By the system $(\star \star)$ there is an element $a \in R$ and an integer $n_{1}$ such that $a+\left(x_{1}^{n_{1}}, \ldots, x_{t}^{n_{1}}\right)$ maps to $\bar{a}$. Let $e$ be an integer such that $F^{e}(\bar{a})=0$. Hence the image of $a^{p^{e}}+\left(x_{1}^{n_{1} p^{e}}, \ldots, x_{t}^{n_{1} p^{e}}\right)$ is the zero in the limit. We can choose an integer $n_{2}>n_{1}$ such that

$$
\varphi_{n_{1} p^{e}, n_{2} p^{e}}\left(a^{p^{e}}+\left(x_{1}^{n_{1} p^{e}}, \ldots, x_{t}^{n_{1} p^{e}}\right)\right)=0 \in R /\left(x_{1}^{n_{2} p^{e}}, \ldots, x_{t}^{n_{2} p^{e}}\right) .
$$

Using the commutative diagram $(\star \star)$ we have

$$
F^{e}\left(\varphi_{n_{1}, n_{2}}\left(a+\left(x_{1}^{n_{1}}, \ldots, x_{t}^{n_{1}}\right)\right)\right)=\varphi_{n_{1} p^{e}, n_{2} p^{e}}\left(F^{e}\left(a+\left(x_{1}^{n_{1}}, \ldots, x_{t}^{n_{1}}\right)\right)\right)=0 .
$$

Therefore $\varphi_{n_{1}, n_{2}}\left(a+\left(x_{1}^{n_{1}}, \ldots, x_{t}^{n_{1}}\right)\right) \in\left(x_{1}^{n_{2}}, \ldots, x_{t}^{n_{2}}\right)^{F} /\left(x_{1}^{n_{2}}, \ldots, x_{t}^{n_{2}}\right)$. Moreover this element maps to $\bar{a}$. This completes the proof.

Theorem 3.4. Let $(R, \mathfrak{m})$ be a local ring of positive characteristic $p>0$ of dimension $d$. Then Fte $(R) \geq H S L(R)$.

Proof. There is nothing to do if $F t e(R)=\infty$. Therefore we can assume henceforth that $F t e(R)=e_{0}$ a finite number. By the prime avoidance theorem we can choose a system of parameters $x_{1}, \ldots, x_{d}$ of $R$ that is also a filter regular sequence. For all $t \leq d$ and all $n \geq 1$ we have

$$
\begin{aligned}
\left(\left(x_{1}^{n}, \ldots, x_{t}^{n}\right)^{F}\right)^{\left[p^{e_{0}}\right]} & \subseteq \bigcap_{m \geq 1}\left(\left(x_{1}^{n}, \ldots, x_{t}^{n}, x_{t+1}^{m}, \ldots, x_{d}^{m}\right)^{F}\right)^{\left[p^{e_{0}}\right]} \\
& =\bigcap_{m \geq 1}\left(x_{1}^{n}, \ldots, x_{t}^{n}, x_{t+1}^{m}, \ldots, x_{d}^{m}\right)^{\left[p^{e_{0}}\right]} \\
& =\left(x_{1}^{n}, \ldots, x_{t}^{n}\right)^{\left[p^{e_{0}}\right]},
\end{aligned}
$$

where the first equation follows from the definition of Frobenius test exponent, and the second equation follows from Krull's intersection theorem. Hence

$$
\left(\left(x_{1}^{n}, \ldots, x_{t}^{n}\right)^{F}\right)^{\left[p^{e} 0\right]}=\left(x_{1}^{n}, \ldots, x_{t}^{n}\right)^{\left[p^{e} 0\right]}
$$

for all $t \leq d$ and for all $n \geq 1$. By Proposition 3.3 we have

$$
0_{H_{\left(x_{1}, \ldots, x_{t}\right)}^{F}}^{t}(R) \cong \underset{n}{\lim } \frac{\left(x_{1}^{n}, \ldots, x_{t}^{n}\right)^{F}}{\left(x_{1}^{n}, \ldots, x_{t}^{n}\right)} .
$$


Following the above observation we have

$$
\frac{\left(x_{1}^{n}, \ldots, x_{t}^{n}\right)^{F}}{\left(x_{1}^{n}, \ldots, x_{t}^{n}\right)} \stackrel{F^{e_{0}}}{\longrightarrow} \frac{\left(x_{1}^{n p^{e_{0}}}, \ldots, x_{t}^{n p^{e_{0}}}\right)^{F}}{\left(x_{1}^{n p^{e_{0}}}, \ldots, x_{t}^{n p^{e_{0}}}\right)}
$$

is the zero map for all $n \geq 1$ and for all $t \leq d$, and so are the limit maps. Therefore $F^{e_{0}}\left(0_{H_{\left(x_{1}, \ldots, x_{t}\right)}^{F}(R)}\right)=0$ for all $t \leq d$. On the other hand by the Nagel-Schenzel theorem we have $H_{\mathfrak{m}}^{t}(R) \cong H_{\mathfrak{m}}^{0}\left(H_{\left(x_{1}, \ldots, x_{t}\right)}^{t}(R)\right)$. Thus we can consider $H_{\mathfrak{m}}^{t}(R)$ as a submodule of $H_{\left(x_{1}, \ldots, x_{t}\right)}^{t}(R)$ that is compatible with Frobenius actions. Therefore $F^{e_{0}}\left(0_{H_{\mathfrak{m}}^{t}(R)}^{F}\right)=0$ for all $t \leq d$, that is $H S L(R) \leq e_{0}$. The proof is complete.

We next show that the Frobenius test exponent has a good behavior under localization.

Proposition 3.5. Let $(R, \mathfrak{m})$ be a local ring of positive characteristic $p>0$ of dimension $d$. Then Fte $(R) \geq \operatorname{Fte}\left(R_{\mathfrak{p}}\right)$ for all $\mathfrak{p} \in \operatorname{Spec}(R)$.

Proof. We can assume that $F t e(R)=e_{0}$ a finite number. Let $t=\operatorname{ht}(\mathfrak{p})$, and $I=\left(a_{1}, \ldots, a_{t}\right) R_{\mathfrak{p}}$ any parameter ideal of $R_{\mathfrak{p}}$. Following the proof of [13, Proposition 6.9] we can choose a part of system of parameters $x_{1}, \ldots, x_{t}$ of $R$ such that $I=\left(x_{1}, \ldots, x_{t}\right) R_{\mathfrak{p}}$. Extending $x_{1}, \ldots, x_{t}$ to a full system of parameters $x_{1}, \ldots, x_{d}$ of $R$. We have

$$
\begin{aligned}
\left(\left(x_{1}, \ldots, x_{t}\right)^{F}\right)^{\left[p^{e_{0}}\right]} & \subseteq \bigcap_{m \geq 1}\left(\left(x_{1}, \ldots, x_{t}, x_{t+1}^{m}, \ldots, x_{d}^{m}\right)^{F}\right)^{\left[p^{e_{0}}\right]} \\
& =\bigcap_{m \geq 1}\left(x_{1}, \ldots, x_{t}, x_{t+1}^{m}, \ldots, x_{d}^{m}\right)^{\left[p^{e_{0}}\right]} \\
& =\left(x_{1}, \ldots, x_{t}\right)^{\left[p^{e_{0}}\right]} .
\end{aligned}
$$

Thus $\left(\left(x_{1}, \ldots, x_{t}\right)^{F}\right)^{\left[p^{e_{0}}\right]}=\left(x_{1}, \ldots, x_{t}\right)^{\left[p^{e_{0}}\right]}$. Since Frobenius closure commutes with localization (see [13, Lemma 3.3]) we have

$$
\begin{aligned}
\left(I^{F}\right)^{\left[p^{\left.e_{0}\right]}\right]} & =\left(\left(\left(x_{1}, \ldots, x_{t}\right) R_{\mathfrak{p}}\right)^{F}\right)^{\left[p^{e_{0}}\right]} \\
& =\left(\left(x_{1}, \ldots, x_{t}\right)^{F} R_{\mathfrak{p}}\right)^{\left[p^{e_{0}}\right]} \\
& =\left(\left(x_{1}, \ldots, x_{t}\right)^{F}\right)^{\left[p^{e_{0}}\right]} R_{\mathfrak{p}} \\
& =\left(x_{1}, \ldots, x_{t}\right)^{\left[p^{e_{0}}\right]} R_{\mathfrak{p}} \\
& =I^{\left[p^{e_{0}}\right]}
\end{aligned}
$$

Therefore $F t e\left(R_{\mathfrak{p}}\right) \leq e_{0}$. The proof is complete.

Recall the a function $f: X \rightarrow \mathbb{R} \cup\{\infty\}$, where $X$ is a topological space, is called upper semi-continuous if for any $t \in \mathbb{R} \cup\{\infty\}$ we have $\{x \mid f(x)<t\}$ is an open set of $X$. We close this note with the following natural question, see [11] for the upper semi-continuity of function $H S L: \operatorname{Spec}(R) \rightarrow \mathbb{R} \cup\{\infty\}, \mathfrak{p} \mapsto H L S\left(R_{\mathfrak{p}}\right)$.

Question 2. Is the function Fte $: \operatorname{Spec}(R) \rightarrow \mathbb{R} \cup\{\infty\}, \mathfrak{p} \mapsto F t e\left(R_{\mathfrak{p}}\right)$, upper semicontinuous?

Acknowledgement. The authors are grateful to the referee for careful reading of the paper and valuable suggestions and comments. 


\section{REFERENCES}

[1] J. Asadollahi and P. Schenzel, Some results on associated primes of local cohomology modules, Japanese J. Mathematics 29 (2003), 285-296.

[2] H. Brenner, Bounds for test exponents, Compos. Math. 142 (2006), 451-463.

[3] M. Brodmann and R.Y. Sharp, Local cohomology: an algebraic introduction with geometric applications, Cambridge University Press, 1998.

[4] H. Dao and P.H. Quy, On the associated primes of local cohomology, Nagoya Math. J., to appear.

[5] R. Hartshorne and R. Speiser, Local cohomological dimension in characteristicp, Ann. of Math. 105 (1977), 45-79.

[6] C. Huneke, Tight closure and its applications, CBMS Lecture Notes in Mathematics, Vol.88, Amer. Math. Soc., Providence, (1996).

[7] C. Huneke, M. Katzman, R.Y. Sharp and Y. Yao, Frobenius test exponents for parameter ideals in generalized Cohen-Macaulay local rings, J. Algebra 305 (2006), 516-539.

[8] M. Katzman and R.Y. Sharp, Uniform behaviour of the Frobenius closures of ideals generated by regular sequences, J. Algebra 295 (2006) 231-246.

[9] G. Lyubeznik, F-modules: applications to local cohomology and D-modules in characteristic $p>0$, J. reine angew. Math. 491 (1997), 65-130.

[10] U. Nagel and P. Schenzel, Cohomological annihilators and Castelnuovo-Mumford regularity, in Commutative algebra: Syzygies, multiplicities, and birational algebra, Contemp. Math. 159 (1994), Amer. Math. Soc. Providence, R.I., 307-328.

[11] S. Murru, On the upper semi-continuity of HSL numbers, preprint, arXiv:1302.1124,

[12] T. Polstra and P.H. Quy, Nilpotence of Frobenius actions on local cohomology and Frobenius closure of ideals, preprint, arXiv:1803.04081.

[13] P.H. Quy and K. Shimomoto, F-injectivity and Frobenius closure of ideals in Noetherian rings of characteristic $p>0$, Adv. Math. 313 (2017), 127-166.

[14] P.H. Quy, On the uniform bound of Frobenius test exponents, preprint, arXiv:1804.01012.

[15] R.Y. Sharp, On the Hartshorne-Speiser-Lyubeznik theorem about Artinian modules with a Frobenius action, Proc. Amer. Math. Soc. 135 (2007), 665-670.

Department of Mathematics, Thang Long University, Hanoi, Vietnam

E-mail address: duonghuongtlu@gmail.com

Department of Mathematics, FPt University, Hanoi, Vietnam

E-mail address: quyph@fe.edu.vn 